\title{
HYPERELLIPTIC PARAMETRISATION OF THE GENERALISED ORDER PARAMETER OF THE $N=3$ CHIRAL POTTS MODEL
}

\author{
R. J. BAXTER '
}

(Received 7 December, 2005)

\begin{abstract}
It has been known for some time that the Boltzmann weights of the chiral Potts model can be parametrised in terms of hyperelliptic functions. but as yet no such parametrisation has been applied to the partition and correlation functions. Here we show that for $N=3$ the function $S\left(t_{p}\right)$ that occurs in the recent calculation of the order parameters can be expressed quite simply in terms of such a parametrisation.
\end{abstract}

2000 Mathematics subject classification: 82B05.

Keywords and phrases: statistical mechanics, lattice models, order parameters, chiral Potts model.

\section{Introduction}

There are a few two-dimensional models (and even fewer three-dimensional models) in equilibrium statistical mechanics that have been solved exactly. These are lattice models where spins $\sigma_{i}$ are assigned to the sites $i$ of a lattice (usually the square lattice). Each spin takes one of $N$ possible values and spins $\sigma_{i}, \sigma_{j}$ on adjacent sites $i, j$ interact with a specified positive real Boltzmann weight function $W\left(\sigma_{i}, \sigma_{i}\right)$. One wants to calculate the partition function (also called the sum-over-states)

$$
Z=\sum \prod_{(i, j)} W\left(\sigma_{j}, \sigma_{j}\right)
$$

where the sum is over all states of all the spins, and the product is over all edges $(i, j)$ of the lattice.

If the number of sites is $M$, we expect the limit

$$
\kappa=\lim _{M \rightarrow x} Z^{1 / M}
$$

\footnotetext{
'Mathematical Sciences Institute, The Australian National University. Canberra. ACT 0200, Australia. (C) Australian Mathematical Society 2006, Serial-fee code 1446-1811/06
} 
to exist and to be independent of the shape of the lattice, provided it becomes large in all directions: this is the "thermodynamic limit", and $\kappa$ is the exponential of the free energy per site. If $1, \ldots, m$ are sites fixed on the lattice and the limit is taken so they become infinitely deep within it, then we also expect the average

$$
\left\langle f\left(\sigma_{1}, \ldots, \sigma_{m}\right)\right\rangle=Z^{-1} \sum \prod_{(i j)} f\left(\sigma_{1}, \ldots, \sigma_{m}\right) W\left(\sigma_{j}, \sigma_{j}\right)
$$

to tend to a limit, for any given function $f$ of these $m$ spins.

Because spins only interact with their neighbours, one can build up the lattice one row at a time, and associate a row-to-row "transfer matrix" with such an operation.

To solve such a model, typically one shows that the Boltzmann weights $W$ satisfy the star-triangle or "Yang-Baxter" relations [5]. These ensure certain commutation relations between the transfer matrices, and this is usually a first step towards calculating $\kappa$.

The next step is to calculate the order parameters, which are averages of certain functions of a single spin $\sigma_{1}$ deep within the lattice. This is a harder problem than calculating $\kappa$. For instance, Onsager [19] calculated $\kappa$ for the square-lattice Ising model in 1944, but it was not till 1949 that he announced at a conference his result for the order parameter (namely the spontaneous magnetisation), and not till 1952 before a proof of the result was published by Yang [20] .

However, since then the "corner transfer matrix" method has been developed by Baxter [4], and the "broken rapidity line method" by Jimbo, Miwa and Nakayashiki [18]. For many of the solved models (those with the "rapidity difference" property), these methods make the calculation of the order parameters comparatively straighforward.

Even so, one model has proved challenging, namely the chiral Potts model. This is an $N$-state model where $W\left(\sigma_{j}, \sigma_{j}\right)$ depends only on the spin difference $\sigma_{j}-\sigma_{j}$, $\bmod N$. The Boltzmann weights also depend on two parameters $p, q$, (known as "rapidities"), and on given positive real constants $k, k^{\prime}$, related by

$$
k^{2}+k^{\prime 2}=1
$$

The parameter $k^{\prime}$ plays the role of a temperature, being small at low temperatures. For $0<k^{\prime}<1$ the system displays spontaneous ferromagnetic order, becoming critical as $k^{\prime} \rightarrow 1$.

Its order parameters can be taken to be

$$
\mathcal{M}_{r}=\left\langle\omega^{r r_{1}}\right\rangle \text {, }
$$

where $\omega=\exp (2 \pi \mathrm{i} / N)$ and $r=1, \ldots, N-1$. It was shown in 1988 that its Boltzmann weights satisfy the star-triangle relation [2, 17], and the partition function per site $\kappa$ was soon calculated $[6,7]$. 
The order parameters were another story. The model had developed from a onedimensional quantum spin chain, which has the same order parameters. From series expansions it was conjectured [1] in 1989 that

$$
\mathcal{M}_{r}=k^{r(N-r) / N^{2}} \text {. }
$$

Much effort was expended in the ensuing years (certainly by the author) in attempting to derive this result. It was not until 2005 that this was done $[14,15]$.

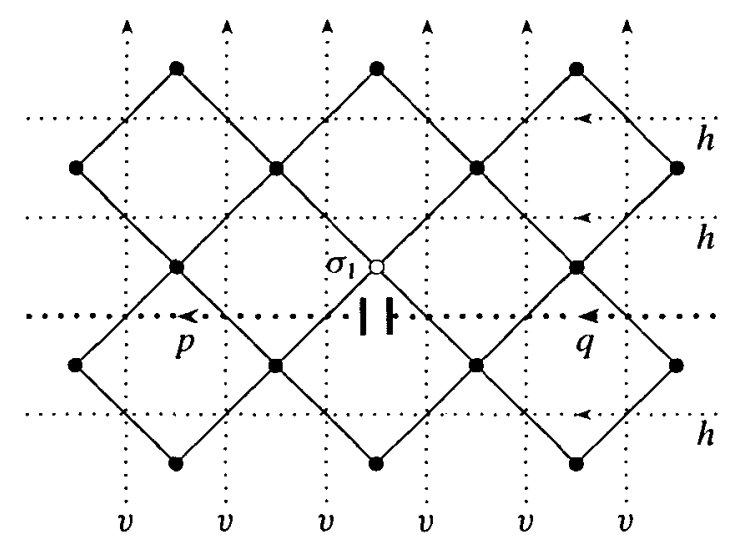

FIGURE 1. The square lattice (circles and solid lines, drawn diagonally) and its medial graph of dotted or broken lines.

The method used was based on that of Jimbo et al. [18]. In Figure 1 we show the square lattice $\mathcal{L}$, drawn diagonally, denoting the sites by circles and the edges by solid lines. We also show as dotted (or broken) lines the medial graph of $\mathcal{L}$. Every edge of $\mathcal{L}$ is intersected by two dotted lines. With each dotted line we associate a rapidity variable $(p, q, h$ or $v)$. In general these variables may differ from dotted line to dotted line. They must be the same all along the line, except for the horizontal broken line immediately below the central spin $\sigma_{1}$. We break this below $\sigma_{1}$ and assign a rapidity $p$ to the left of the break and a rapidity $q$ to the right. With these choices of rapidities, define

$$
\tilde{F}_{p q}(r)=\left\langle\omega^{r \sigma_{1}}\right\rangle
$$

In the thermodynamic limit, the star-triangle relations will ensure that $\tilde{F}_{p q}(r)$ is independent of the "background" rapidities $v, h$, because it allows us to move any of these dotted lines off to infinity [3]. However, the effect of the break is that we cannot move the broken line $p, q$ away from $\sigma_{1}$, so $\tilde{F}_{p q}(r)$ will indeed depend on $p$ and $q$.

An important special case is when $q=p$. Then the $p, q$ rapidity line is not in fact broken, so it can be removed to infinity and $\tilde{F}_{p p}(r)$ must be independent of $p$ and equal to the order parameter $\mathcal{M}_{r}$ defined by (1.2): $\mathcal{M}_{r}=\tilde{F}_{p p}(r)$. 
We also define

$$
G_{p q}(r)=\frac{\tilde{F}_{p q}(r)}{\tilde{F}_{p q}(r-1)} .
$$

The author wrote down [11] functional relations satisfied by $G_{p q}(r)$ in 1998. They do not completely specify $G_{p q}(r)$, but must be supplemented by information on the analyticity properties of $G_{p q}(r)$. (Just as the relation $f(z+1)=f(z)$ only tells us that $f(z)$ is periodic of period 1: however, if we can also show that $f(z)$ is analytic and bounded in the domain $0 \leq \operatorname{Re}(z) \leq 1$, then it follows from Liouville's theorem that $f(z)$ is a constant.)

For $N=2$ the chiral Potts model reduces to the Ising model and it is quite easy to find the needed analyticity information, to solve the functional relations and obtain the Onsager-Yang result $\mathcal{M}_{1}=k^{1 / 4}$.

For $N>2$ the problem is much harder. It was not until late 2004 that the author realised that it is not actually necessary to solve for the general function $G_{p q}(r)$. It is sufficient to do so for a special "superintegrable" case where $q$ is related to $p$. The function then has quite simple analyticity properties and it's quite easy to solve the relations (in fact one does not even need all the relations), to obtain $G_{p q}(r)$ for this case and to verify the 16-year old conjecture (1.3). For $r=1, \ldots, N$, the functions $G_{p q}(r)$ can all be expressed in terms of a single function $S\left(t_{p}\right)$ which is defined below.

Even so, it would still be interesting to understand $G_{p q}(r)$ more generally. A fundamental difficulty is that for $N>2$ the rapidities $p$ and $q$ are points on an algebraic curve of genus greater than 2, and there is no explicit parametrisation of this curve in terms of single-valued functions of a single variable. (There is for $N=2$ : one can then parametrise in terms of Jacobi elliptic functions.) One can parametrise in terms of hyperelliptic functions [8], but these have $N-1$ arguments that are related to one another. As yet they have not proved particularly useful, but one lives in hope. The function $S\left(t_{p}\right)$ is a simple example of a thermodynamic property of the chiral Potts model, and has the simplifying feature that it depends on only one rapidity, rather than two. It is an interesting question whether it can be simply expressed in terms of these hyperelliptic functions.

For $N=3$ these hyperelliptic functions can be expressed in terms of ordinary Jacobi elliptic functions. One still has two related arguments (here termed $z_{p}$ and $w_{p}$ ), but some of the properties can be expressed as products of Jacobi functions, each with an argument $z_{p}$ or $w_{p}$, or some combination thereof. A number of such results have been obtained [10], [9, pages 568-569].

There are two distinct ways of performing the hyperelliptic parametrisation. In $[8,16]$ we used what we shall herein call the "original" parametrisation. What we report here is that for $N=3$ the function $S\left(t_{p}\right)$ can be expressed quite simply as a product of Jacobi functions of $z_{p}$ and $w_{p}$, provided we use the second "alternative" 
parametrisation.

\section{The function $S\left(t_{p}\right)$}

We can take a rapidity $p$ to be a set of variables $p=\left\{x_{p}, y_{p}, \mu_{p}, t_{p}\right\}$ related to one another by

$$
t_{p}=x_{p} y_{p}, \quad x_{p}^{N}+y_{p}^{N}=k\left(1+x_{p}^{N} y_{p}^{N}\right), \quad k x_{p}^{N}=1-\frac{k^{\prime}}{\mu_{p}^{N}}, \quad k y_{p}^{N}=1-k^{\prime} \mu_{p}^{N} .
$$

There are various automorphisms or maps that take one set $\left\{x_{p}, y_{p}, \mu_{p}, t_{p}\right\}$ to another set satisfying the same relations (2.1). Four that we shall use are:

$$
\begin{aligned}
& R:\left\{x_{R p}, y_{R p}, \mu_{R_{p}}, t_{R p}\right\}=\left\{y_{p}, \omega x_{p}, 1 / \mu_{p}, \omega t_{p}\right\}, \\
& S:\left\{x_{S_{p}}, y_{S_{p}}, \mu_{S_{p}}, t_{S p}\right\}=\left\{y_{p}^{-1}, x_{p}^{-1}, \omega^{-1 / 2} y_{p} /\left(x_{p} \mu_{p}\right), t_{p}^{-1}\right\}, \\
& V:\left\{x_{V_{p}}, y_{V_{p}}, \mu_{V_{p}}, t_{V_{p}}\right\}=\left\{x_{p}, \omega y_{p}, \mu_{p}, \omega t_{p}\right\}, \\
& M:\left\{x_{M p}, y_{M p}, \mu_{M p}, t_{M p}\right\}=\left\{x_{p}, y_{p}, \omega \mu_{p}, t_{p}\right\} .
\end{aligned}
$$

They satisfy

$$
R V^{-1} R=V, \quad M R M=R, \quad M S M=S, \quad S^{2}=V^{N}=M^{N}=1 .
$$

Let $q$ be another rapidity set, related to $p$ by $q=V p$, that is,

$$
x_{q}=x_{p}, \quad y_{q}=\omega y_{p}, \quad \mu_{q}=\mu_{p} .
$$

We take $\mu_{p}$ to be outside the unit circle, so $\left|\mu_{p}\right|>1$. Then we can specify $x_{p}$ uniquely by requiring that $-\pi /(2 N)<\arg \left(x_{p}\right)<\pi /(2 N)$.

We regard $x_{p}, y_{p}, \mu_{p}^{N}$ as functions of $t_{p}$. Then $t_{p}$ lies in a complex plane containing $N$ branch cuts $\mathcal{B}_{0}, \ldots, \mathcal{B}_{N-1}$ on the lines $\arg \left(t_{p}\right)=0,2 \pi / N, \ldots, 2 \pi(N-1) / N$, as indicated in Figure 2, while $x_{p}$ lies in a near-circular region round the point $x_{p}=1$, as indicated schematically by the region $\mathcal{R}_{0}$ inside the dotted curve of Figure 2 . The variable $y_{p}$ can lie anywhere in the complex plane except in $\mathcal{R}_{0}$ and in $N-1$ corresponding near-circular regions $\mathcal{R}_{1}, \ldots, \mathcal{R}_{N-1}$ round the other branch cuts. With these choices, we say that $p$ lies in the "domain" $\mathcal{D}$.

With these choices, we show in [15] that

$$
G_{p q}(r)=k^{(N+1-2 r) / N^{2}} S\left(t_{p}\right)
$$

for $r=1, \ldots, N-1$, while $G_{p q}(0)=G_{p q}(N)=k^{(1-N) / N^{2}} S\left(t_{p}\right)^{1-N}$. Hence $G_{p q}(1) \cdots G_{p q}(N)=1$, in agreement with the definition (1.4). The function $S_{p}=$ $S\left(t_{p}\right)$ is given by

$$
\log S\left(t_{\rho}\right)=-\frac{2}{N^{2}} \log k+\frac{1}{2 N \pi} \int_{0}^{2 \pi} \frac{k^{\prime} \mathrm{e}^{\mathrm{i} \theta}}{1-k^{\prime} \mathrm{e}^{\mathrm{i} \theta}} \log \left[\Delta(\theta)-t_{p}\right] \mathrm{d} \theta,
$$




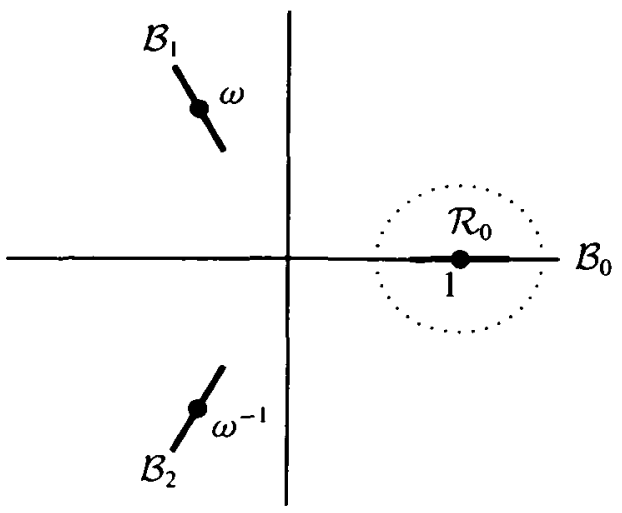

FIGURE 2. The cut $t_{p}$-plane for $N=3$, showing the three branch cuts $\mathcal{B}_{0}, \mathcal{B}_{1}, \mathcal{B}_{2}$ and the approximately circular region $\mathcal{R}_{0}$ in which $x_{p}$ lies when $p \in \mathcal{D}$.

where $\Delta(\theta)=\left[\left(1-2 k^{\prime} \cos \theta+k^{\prime 2}\right) / k^{2}\right]^{1 / N}$.

From [14], particular properties are

$$
S(0)=1, \quad S(\infty)=k^{-2 / N^{2}}, \quad S\left(t_{p}\right) S\left(\omega t_{p}\right) \cdots S\left(\omega^{N-1} t_{p}\right)=k^{-1 / N} x_{p} .
$$

The function $S\left(t_{p}\right)$ is single-valued, non-zero and analytic in the cut $t_{p}$ plane of Figure 2, but only the cut on the positive real axis is necessary: the other cuts can be removed for this function. If $S_{a c}\left(t_{p}\right)$ is the analytic continuation of $S\left(t_{p}\right)$ across the branch cut $\mathcal{B}_{r}$, then

$$
S_{a c}\left(t_{p}\right)= \begin{cases}S\left(t_{p}\right) & \text { for } r \neq 0 \\ \left(y_{p} / x_{p}\right) S\left(t_{p}\right) & \text { for } r=0 .\end{cases}
$$

If we interchange $p, q$ in [15, Equation 49], then apply the restriction (2.4) and use the relation $R S=M V R S V$ together with [15, Equation 50], we obtain

$$
G_{p q}(r) G_{p^{\prime} \cdot q^{\prime}}(N-r)=1,
$$

where $p^{\prime}=V^{-1} q^{\prime}=R S V p$. It follows that $S\left(t_{p}\right)$ also has the symmetry

$$
S_{p} S_{R S V_{p}}=S\left(t_{p}\right) S\left(1 / t_{p}\right)=k^{-2 / N^{2}} .
$$

\section{The Riemann sheets (“domains”) formed by analytic continuation}

We shall want to consider the analytic continuation of certain functions of $t_{p}$, onto other Riemann sheets, that is, beyond the domain $\mathcal{D}$. We restrict attention to functions 
that are meromorphic and single-valued in the cut plane of Figure 2, and similarly for their analytic continuations. Obvious examples are $x_{p}, y_{p}$ and $S\left(t_{p}\right)$. They are therefore meromorphic and single-valued on their Riemann surfaces, but we need to know what these surfaces are.

We start by considering the most general such surface. As a first step, allow $\mu_{p}$ to move from outside the unit circle to inside. Then $t_{p}$ will cross one of the $N$ branch cuts $\mathcal{B}_{i}$ in Figure 2, moving onto another Riemann sheet, going back to its original value but now with $y_{p}$ in $\mathcal{R}_{i}$. Since $y_{p}$ is thereby confined to the region near and surrounding $\omega^{i}$, we say that $y_{p} \simeq \omega^{i}$. Conversely, by $y_{p} \simeq \omega^{i}$ we mean that $y_{p} \in \mathcal{R}_{i}$.

We say that $p$ has moved into the domain $\mathcal{D}_{i}$ adjacent to $\mathcal{D}$. There are $N$ such domains $\mathcal{D}_{0}, \mathcal{D}_{1}, \ldots, \mathcal{D}_{N-1}$.

Now allow $\mu_{p}$ to become larger than one, so $t_{p}$ again crosses one of the $N$ branch cuts. Again we require that $t_{p}$ returns to its original value. If it crosses $\mathcal{B}_{i}$, then it moves back to the original domain $\mathcal{D}$. However, if it crosses another cut $\mathcal{B}_{j}$ then $x_{p}$ moves into $\mathcal{R}_{j-i}$, and we say that $p$ is now in domain $\mathcal{D}_{i, j-i}$.

Proceeding in this way, we build up a Cayley tree of domains. For instance, the domain $\mathcal{D}_{i j k}$ is a third neighbour of $\mathcal{D}$, linked via the first neighbour $\mathcal{D}_{i}$ and the second-neighbour $\mathcal{D}_{i j}$, as indicated in Figure 3. Here $x_{p} \simeq 1$ in $\mathcal{D}, y_{p} \simeq \omega^{i}$ in $\mathcal{D}_{i}$, $x_{p} \simeq \omega^{j}$ in $\mathcal{D}_{i j}$ and $y_{p} \simeq \omega^{k}$ in $\mathcal{D}_{i j k}$. We reject moves that take $p$ back to the domain immediately before the last, so $j \neq 0$ and $k \neq i$. We refer to the sequence $\{i, j, k, \ldots\}$ that define any domain as a route. We can think of it as a sequence of points, all with the same value of $t_{p}$, on the successive Riemann sheets or domains.

The domains $\mathcal{D}, \mathcal{D}_{i j}, \mathcal{D}_{i j k \ell}, \ldots$ with an even number of indices, have $x \simeq \omega^{\ell}$, where $\ell$ is the last index. We refer to them as being of even parity and of type $\ell$. The domains $\mathcal{D}_{i}, \mathcal{D}_{i j k}, \ldots$ have $y \simeq \omega^{\ell}$ and are of odd parity and type $\ell$.

$$
\mathcal{D}-\mathcal{D}_{i} \longrightarrow \mathcal{D}_{i j} \longrightarrow \mathcal{D}_{i j k}
$$

FIGURE 3. A sequence of adjacent domains $\mathcal{D}, \mathcal{D}_{i}, \mathcal{D}_{i j}, \mathcal{D}_{i j k}$.

The automorphism that takes a point $p$ in $\mathcal{D}$ to a point in $\mathcal{D}_{i}$, respectively, is the mapping $A_{i}=V^{i-1} R V^{-i}$. If $q=A_{i} p$, then

$$
x_{q}=\omega^{-i} y_{p}, \quad y_{q}=\omega^{i} x_{p}, \quad t_{q}=t_{p} .
$$

Because of (2.3), $A_{i+N}=A_{i}$, so there are $N$ such automorphisms.

We can use these maps to generate all the sheets in the full Cayley tree. Suppose we have a domain with route $\{i, j, k, \ldots\}$ and we apply the automorphism $A_{\alpha}$ to all points on the route. From (3.1) this will generate a new route $\{\alpha, i-\alpha, j+\alpha, k-\alpha, \ldots\}$. For instance, if we apply the map $A_{\alpha}$ to the route $\{m\}$ from $\mathcal{D}$ to $\mathcal{D}_{m}$, we obtain the route $\{\alpha, m-\alpha\}$ to the domain $D_{\alpha, m-\alpha}$. Thus the map that takes $\mathcal{D}$ to $\mathcal{D}_{i j}$ is $A_{i} A_{i+j}$. 
Iterating, we find that the map that takes $\mathcal{D}$ to $\mathcal{D}_{i j k \ldots m n}$ is

$$
A_{i} A_{i+j} A_{j+k} \cdots A_{m+n} .
$$

We must have

$$
A_{i}^{2}=1
$$

since applying the same map twice merely returns $p$ to the previous domain.

Let us refer to the general Riemann surface we have just described as $\mathcal{G}$. It consists of infinitely many Riemann sheets, each sheet corresponding to a site on a Cayley tree, adjacent sheets corresponding to adjacent points on the tree. A Cayley tree is a huge graph: it contains no circuits and is infinitely dimensional, needing infinitely many integers to specify all its sites.

Any given function will have a Riemann surface that can be obtained from $\mathcal{G}$ by identifying certain sites with one another, thereby creating circuits and usually reducing the graph to one of finite dimensionality.

From (3.1), the maps $A_{0}, A_{1}, \ldots, A_{N-1}$ leave $t_{p}$ unchanged. We shall often find it helpful to regard $t_{p}$ as a fixed complex number, the same in all domains, and to consider the corresponding values of $x_{p}, y_{p}$ (and the hyperelliptic variables $z_{p}, w_{p}$ ) in the various domains. To within factors of $\omega$, the variables $x_{p}$ and $y_{p}$ will be the same as those for $\mathcal{D}$ in even domains, while they will be interchanged on odd domains.

Analytic continuation of $S\left(t_{p}\right) \quad$ Now return to considering the function $S\left(t_{p}\right)$. It is sometimes helpful to write this more explicitly as $S\left(x_{p}, y_{p}\right)$. Then from (2.8) the map that takes $S\left(t_{p}\right)$ from domain $\mathcal{D}$ to $\mathcal{D}_{i}$ is

$$
q=A_{i} p: \quad S\left(x_{q}, y_{q}\right)=\left(y_{q} / x_{q}\right)^{-\delta_{i}} S\left(x_{p}, y_{p}\right),
$$

where $x_{q}, y_{q}$ are given by (3.1) and $\delta_{i}=1$ if $i=0, \bmod N$; otherwise $\delta_{i}=0$. Note that $x_{q}, y_{q}$ are obtained by interchanging $x_{p}, y_{p}$ and multiplying them by powers of $\omega$.

For given $t_{p}$, let $S_{0}\left(t_{p}\right)$ be the value of $S\left(t_{p}\right)$ in the central domain $\mathcal{D}$, given by the formula (2.6). Iterating the mappings (3.4) from domain to domain, in any domain we must have

$$
S\left(x_{p}, y_{p}\right)=\omega^{\alpha}\left(y_{p} / x_{p}\right)^{r} S_{0}\left(t_{p}\right),
$$

where $\alpha, r$ are integers. Note that in this equation $x_{p}, y_{p}$ are the values for the domain being considered: they are not the corresponding initial values of the central domain $\mathcal{D}$.

In particular, in the domain $\mathcal{D}_{i j k}$ we obtain

$$
r=-\delta_{i}+\delta_{i+j}-\delta_{j+k}
$$




\section{The original hyperelliptic parametrisation for $N=3$}

Hereinafter we restrict our attention to the case $N=3$ and use the hyperelliptic parametrisation and notation of previous papers $[8-10,12]$. We use only formulae that involve ordinary Jacobi elliptic (or similar) functions of one variable.

Given $k, k^{\prime}$, we define a "nome" $x$ by

$$
\left(k^{\prime} / k\right)^{2}=27 x \prod_{n=1}^{\infty}\left(\frac{1-x^{3 n}}{1-x^{n}}\right)^{12} .
$$

We regard $x$ as a given constant, not the same as the rapidity variable $x_{p}$. It is small at low temperatures $\left(k^{\prime}\right.$ small), and increases to unity at criticality $\left(k^{\prime}=1\right)$. We introduce two elliptic-type functions

$$
\begin{aligned}
& h(z)=\omega^{2} h(x z)=\prod_{n=1}^{\infty} \frac{\left(1-\omega x^{n-1} z\right)\left(1-\omega^{2} x^{n} / z\right)}{\left(1-\omega^{2} x^{n-1} z\right)\left(1-\omega x^{n} / z\right)}, \\
& \phi(z)=z^{1 / 3} \prod_{n=1}^{\infty} \frac{\left(1-x^{3 n-2} / z\right)\left(1-x^{3 n-1} z\right)}{\left(1-x^{3 n-2} z\right)\left(1-x^{3 n-1} / z\right)} .
\end{aligned}
$$

We then define two further variables $z_{p}, w_{p}$ by

$$
t_{p}=x_{p} y_{p}=\omega h\left(z_{p}\right)=h\left(-1 / w_{p}\right)=\omega^{2} h\left(-w_{p} / z_{p}\right) .
$$

These are the relations (27) of [10]. The relations (32) of [10] are also satisfied:

$$
x_{p}^{-3} y_{p}^{3} \mu_{p}^{-6}=\phi\left(x z_{p} / w_{p}^{2}\right)^{3}=\phi\left(-x z_{p} w_{p}\right)^{3}=\phi\left(-x w_{p} / z_{p}^{2}\right)^{3},
$$

as are the relations (4.5), (4.6) of [9], in particular,

$$
w=\prod_{n=1}^{\infty} \frac{\left(1-x^{2 n-1} z / w\right)\left(1-x^{2 n-1} w / z\right)\left(1-x^{6 n-5} z w\right)\left(1-x^{6 n-1} z^{-1} w^{-1}\right)}{\left(1-x^{2 n-2} z / w\right)\left(1-x^{2 n} w / z\right)\left(1-x^{6 n-2} z w\right)\left(1-x^{6 n-4} z^{-1} w^{-1}\right)}
$$

writing $z_{p}, w_{p}$ here simply as $z, w$.

The $z_{p}, w_{p}$ variables satisfy the automorphisms

$$
\begin{aligned}
& z_{R_{p}}=x z_{p}, \quad z_{S_{p}}=1 /\left(x z_{p}\right), \quad z_{v_{p}}=-1 / w_{p}, \\
& w_{R p}=z_{p} / w_{p}, \quad w_{S p}=1 /\left(x w_{p}\right), \quad w_{v_{p}}=z_{p} / w_{p} .
\end{aligned}
$$

The operation $p \rightarrow M p$ multiplies $\left(z_{p} w_{p}\right)^{1 / 3}$ by $\omega$, but does not change $z_{p}, w_{p}$ themselves.

The variables $z_{p}, w_{p}$ are of order unity when $k^{\prime}, x$ are small, $\mu_{p}$ is of order $1 / k^{\prime}$, and $x_{p} \simeq 1$. This is the low-temperature limiting case of $p \in \mathcal{D}$. It is convenient to define $u_{p}=\left\{z_{p},-1 / w_{p},-w_{p} / z_{p}\right\}$. 
The three automorphisms that leave $t_{p}$ unchanged, while taking $\mathcal{D}$ to $\mathcal{D}_{0}, \mathcal{D}_{1}, \mathcal{D}_{2}$, respectively, are

$$
A_{0}=V^{2} R, \quad A_{1}=R V^{2}, \quad A_{2}=V R V .
$$

If $q=A_{i} p$, then

$$
x_{q}=\omega^{-i} y_{p}, \quad y_{q}=\omega^{i} x_{p}, \quad t_{q}=t_{p},
$$

and $u_{q}=\mathcal{A}_{i} u_{p}$, where $\mathcal{A}_{0}, \mathcal{A}_{1}, \mathcal{A}_{1}$ are the three-by-three matrices

$$
\mathcal{A}_{0}=\left(\begin{array}{ccc}
0 & x^{-1} & 0 \\
x & 0 & 0 \\
0 & 0 & 1
\end{array}\right), \quad \mathcal{A}_{1}=\left(\begin{array}{ccc}
0 & 0 & x \\
0 & 1 & 0 \\
x^{-1} & 0 & 0
\end{array}\right), \quad \mathcal{A}_{2}=\left(\begin{array}{ccc}
1 & 0 & 0 \\
0 & 0 & x^{-1} \\
0 & x & 0
\end{array}\right)
$$

They satisfy the identities

$$
\mathcal{A}_{i} \mathcal{A}_{j} \mathcal{A}_{i}=\mathcal{A}_{j} \mathcal{A}_{i} \mathcal{A}_{j}
$$

for all $i, j$.

They permute the three elements $z_{p},-1 / w_{p},-w_{p} / z_{p}$ of $u_{p}$ and multiply them by powers of $x$, the product of the elements remaining unity. Let $z_{p}^{0}, w_{p}^{0}$ be the values of $z_{p}, w_{p}$ on the central sheet $\mathcal{D}$. Then it follows that on any sheet, for the same common value of $t_{p}$,

$$
z_{p}=x^{m} \alpha_{p}, \quad w_{p}=x^{n} \beta_{p},
$$

where $\left\{\alpha_{p},-1 / \beta_{p},-\beta_{p} / \alpha_{p}\right\}$ is a permutation of $\left\{z_{p}^{0},-1 / w_{p}^{0},-w_{p}^{0} / z_{p}^{0}\right\}$.

Repeated applications of the three automorphisms will therefore generate a twodimensional set of permutations and multiplications of the elements of $u_{p}$. Each member of the set corresponds to a site on the honeycomb lattice of Figure 4. Adjacent Riemann sheets correspond to adjacent sites of the lattice. Sheets of even parity correspond to sites represented by circles, those of odd parity are represented by squares. If $i$ is the integer inside the circle or square, then for even sites $x_{p} \simeq \omega^{-i}$, while on odd sites $y_{p} \simeq \omega^{i}$. The numbers shown in brackets alongside each site are the integers $m, n$ of (4.11).

Thus for the functions $z_{p}$ and $w_{p}$ of $t_{p}$, the graph $\mathcal{G}$ of the Riemann surface reduces to this two-dimensional honeycomb lattice.

Note that the sites $X, Y, Z$ in the figure are third neighbours of the central site $\mathcal{D}$, and each can be reached from $\mathcal{D}$ in two three-step ways. For instance, $Y$ is both $\mathcal{D}_{021}$ and $\mathcal{D}_{211}$. (Note that for $\mathcal{D}_{i j k}$ we here take the intermediate site $j$ to be represented in Figure 4 by the integer $-j, \bmod 3$. This is changed in the next section to $+j$.)

Thus $Y$ is obtainable from $\mathcal{D}$ by the maps $A_{0} A_{2} A_{0}$ and $A_{2} A_{0} A_{2}$. From (4.10) these are the same, so we can identify the two sheets as one, represented by $Y$. 
Similarly, $X$ corresponds to $A_{1} A_{2} A_{1}=A_{2} A_{1} A_{2}$ and $Z$ to $A_{0} A_{1} A_{0}=A_{1} A_{0} A_{1}$. This is why $\mathcal{G}$ reduces to the honeycomb lattice.

However, the automorphisms (3.4) of the function $S\left(t_{p}\right)$ do not in general satisfy (4.10). On its Riemann sheets $\mathcal{D}_{021}, \mathcal{D}_{211}$ we find from (3.6) that $r=-2$ and 1. Thus from (3.5) the analytic continuation of $S\left(t_{p}\right)$ is $\left(y_{p} / x_{p}\right)^{-2} S_{0}\left(t_{p}\right)$ and $\left(y_{p} / x_{p}\right) S_{0}\left(t_{p}\right)$ on each sheet, respectively (ignoring factors of $\omega$ ). Thus the result for $Y$ depends on the route taken to it. (The same is true of $Z$, but not for $X$.)

Hence the values of $z_{p}$ (and $w_{p}$ ) are the same on $\mathcal{D}_{021}$ and $\mathcal{D}_{211}$, but $S\left(t_{p}\right)$ is different. It follows that $z_{p}, w_{p}$ do not uniquely determine $S\left(t_{p}\right)$. Hence neither $S\left(t_{p}\right)$ nor $S\left(t_{p}\right)^{3}$ is a single-valued function of these hyperelliptic variables $z_{p}, w_{p}$ : one must look elsewhere for such a parametrisation.

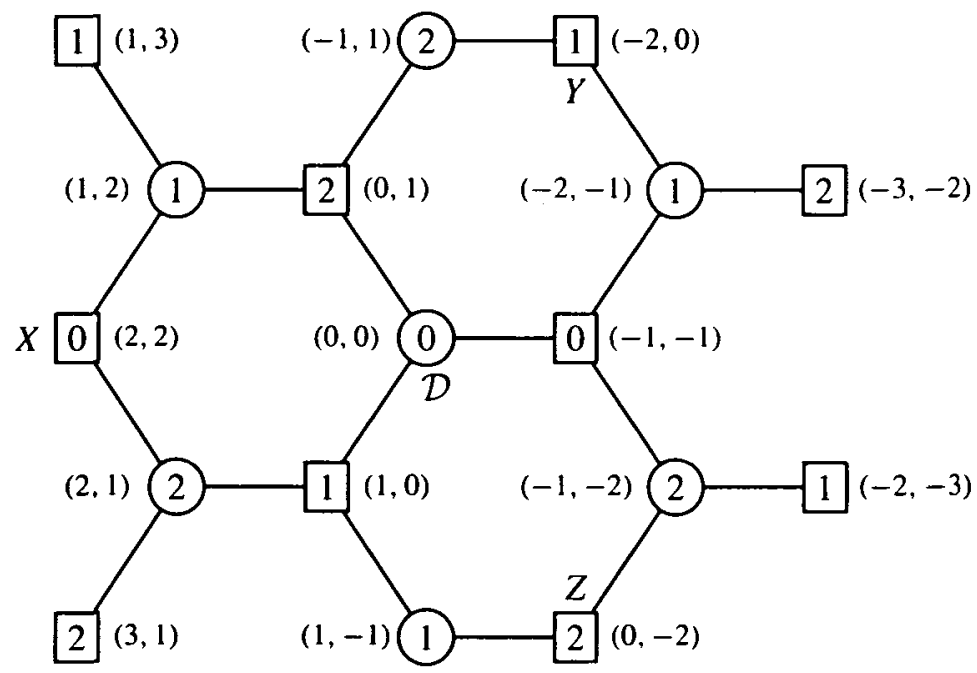

FIGURE 4. The honeycomb lattice formed by the hyperelliptic variables $z, w$ in either parametrisation. Circles (squares) denote sites of even (odd) parity.

\section{The alternative hyperelliptic parametrisation for $N=3$}

There is another way of parametrising $k, x_{p}, y_{p}, \mu_{p}, t_{p}$ so that the nome $x$ is small when $k^{\prime}$ is small. It can be obtained from the original parametrisation of the previous section by a simple mapping, and we do this in the Appendix. We take the results (A.1)-(A.4) therein and drop the hats on $x_{p}, y_{p}, \ldots, V, M$ to obtain

$$
-k^{\prime 2}=27 x \prod_{n=1}^{\infty}\left(\frac{1-x^{3 n}}{1-x^{n}}\right)^{12} \text {. }
$$




$$
\begin{aligned}
y_{p} / x_{p} & =\omega h\left(z_{p}\right)=h\left(-1 / w_{p}\right)=\omega^{2} h\left(-w_{p} / z_{p}\right), \\
-x_{p}^{-3} y_{p}^{3} \mu_{p}^{-6} & =\phi\left(x z_{p} / w_{p}^{2}\right)^{3}=\phi\left(-x z_{p} w_{p}\right)^{3}=\phi\left(-x w_{p} / z_{p}^{2}\right)^{3} . \\
z_{R p} & =-x w_{p}, \quad z_{S p}=-1 /\left(x w_{p}\right), \quad z_{V p}=-1 / w_{p}, \\
w_{R p} & =w_{p} / z_{p}, \quad w_{S p}=-1 /\left(x z_{p}\right), \quad w_{V p}=z_{p} / w_{p} .
\end{aligned}
$$

Again $z_{M p}=z_{p}, w_{M p}=w_{p}$. These equations replace (4.1), (4.4), (4.5), (4.7) of Section 4. The functions $h(z), \phi(z)$ remain defined by (4.2) and (4.3), and the relation (4.6) remains satisfied.

We now regard $x_{p}, y_{p}, \ldots, V, M$ as being the same variables and automorphisms as those above, satisfying (2.1), (2.2) and (2.3). Then the hyperelliptic variables $x, z_{p}, w_{p}$ are different from those of Section 4. If one takes $p \in \mathcal{D}$ and expands the functions in powers of the low-temperature variable $k^{\prime}$, then to leading order $z_{p}, w_{p}$ are the same as the $z_{p}, w_{p}$ of Section 4 , being of order unity and satisfying $w_{p}=z_{p}+1$; $x$ is negated and is of order $k^{\prime}$.

The three automorphisms that leave $t_{p}$ unchanged while taking $\mathcal{D}$ to $\mathcal{D}_{0}, \mathcal{D}_{1}, \mathcal{D}_{2}$ are again given by (4.8) and (4.9). Using the rules (5.4), we find that $z_{p}, w_{p}$ transform according to the rules

$$
q=A_{i} p: \quad z_{q}=x^{2-i-3 \delta(i)} / z_{p}, \quad w_{q}=x^{i-1} / w_{p},
$$

for $i=0,1,2$, writing $\delta_{i}$ as $\delta(i)$. If $z_{p}^{0}, w_{p}^{0}$ are the values of $z_{p}, w_{p}$ on the central sheet $\mathcal{D}$. then it follows that on any Riemann sheet the analytic continuations of $z_{p}, w_{p}$ (for a given value of $t_{p}$ ) are

$$
x^{m}\left(z_{p}^{0}\right)^{ \pm 1}, \quad x^{n}\left(w_{p}^{0}\right)^{ \pm 1}
$$

choosing the upper (lower) signs on sheets of even (odd) parity. Here $m, n$ are integers satisfying

$$
m+n=\left\{\begin{array}{lll}
0 & (\bmod 3) & \text { on even sheets, } \\
1 & (\bmod 3) & \text { on odd sheets. }
\end{array}\right.
$$

The Riemann surface for $z_{p}, w_{p}$ therefore corresponds to a two-dimensional graph $\mathcal{G}$, each site of $\mathcal{G}$ being specified by the two integers $m, n$.

In fact this $\mathcal{G}$ is the honeycomb lattice shown in Figure 4, but we must interpret it slightly differently from how we did in Section 4 . Adjacent sites still correspond to adjacent Riemann sheets, and the parities of the sites are shown as in Section 4 by circles and squares. Again, on odd sites $y_{p} \simeq \omega^{i}$, where $i$ is the number shown in the figure. However, on even sites we now take $x_{p} \simeq \omega^{i}$. For each site, the bracketed integers shown in Figure 4 are now the integers $(m, n)$ of $(5.6)$. 
As in the previous section, the sites $X, Y, Z$ in the figure are third neighbours of the central site $\mathcal{D}$, and each can be reached from $\mathcal{D}$ in two three-step ways. There is the difference that the $j$ of $\mathcal{D}_{i j k}$ is now the number inside the corresponding circle of Figure 4, so in this section $X$ is both $\mathcal{D}_{210}$ and $\mathcal{D}_{120}$, so from (3.2) and (3.3) it is obtainable from $\mathcal{D}$ by the maps $A_{2} A_{0} A_{1}$ and $A_{1} A_{0} A_{2}$. The reason we can identify $\mathcal{D}_{210}$ with $\mathcal{D}_{120}$ is that (5.5) implies that $A_{2} A_{0} A_{1}=A_{1} A_{0} A_{2}$. More generally, it implies that

$$
A_{i} A_{j} A_{k}=A_{k} A_{j} A_{i}
$$

for all permutations $i, j, k$ of $0,1,2$. This means that each $X, Y, Z$ corresponds to a single Riemann sheet rather than two, and is the reason that $\mathcal{G}$ reduces (for the functions $z_{p}, w_{p}$ of $t_{p}$ ) from the full Cayley tree to the honeycomb lattice.

Properties of $z_{p}, w_{p}, S\left(t_{p}\right) \quad$ Within the central domain $\mathcal{D}$ there is some circle of nonzero radius, centre the origin, such that none of $z_{p}, w_{p}, 1 / z_{p}, 1 / w_{p}, z_{p} / w_{p}, w_{p} / z_{p}$ can lie within the circle (for $x$ small the radius is of order $x^{1 / 2}$ ). Two special values of $p$ that lie within $\mathcal{D}$ are

$$
p(1): \quad z_{p}=-\frac{1}{w_{p}}=-\frac{w_{p}}{z_{p}}=\omega^{2}, \quad y_{p}=0, \quad x_{p}=k^{1 / 3}, \quad S\left(t_{p}\right)=1,
$$

and

$$
p(2): \quad z_{p}=-\frac{1}{w_{p}}=-\frac{w_{p}}{z_{p}}=\omega, \quad y_{p}=\infty, \quad x_{p}=k^{-1 / 3}, \quad S\left(t_{p}\right)=k^{-2 / 9},
$$

using (2.1) and (2.7).

Remembering that $x_{p} \simeq 1$, one can verify that the conditions (4.6), (5.2) are satisfied. These are the only two points within $\mathcal{D}$ where $y_{p} / x_{p}$ has a zero or pole.

Any point where one of $z_{p},-1 / w_{p},-w_{p} / z_{p}$ is equal to $\omega^{2} x^{m}$ or $\omega x^{m}$, for non-zero integer $m$, necessarily lies outside $\mathcal{D}$ (that is, on another Riemann sheet). It follows that the functions $G\left(z_{p}\right), \ldots, h\left(-w_{p} / z_{p}\right)$ defined and used in the next section have no zeros or poles for $p \in \mathcal{D}$ : they are finite and non-zero therein. The same is true of $x_{p}$, $S\left(t_{p}\right)$ and the function $\tilde{S}\left(z_{p}, w_{p}\right)$ defined below.

Now consider the maps $A_{i}$ for the function $S\left(t_{p}\right)$, as given in (3.4)-(3.6). The mapping $A_{i} A_{j} A_{k}$ simply multiplies $S\left(t_{p}\right)$ by a power of $\omega$ and by $\left(y_{p} / x_{p}\right)^{r}$, where $r=-\delta_{i}+\delta_{j}-\delta_{k}$. Obviously this exponent $r$ is unchanged by interchanging $i$ with $k$, so is the same for both sides of (5.7).

The powers of $\omega$ are not necessarily the same, but we can avoid this difficulty by simply working with $S\left(t_{p}\right)^{3}$ instead of $S\left(t_{p}\right)$. The automorphisms $A_{i}$ for $S\left(t_{p}\right)^{3}$ do satisfy (5.7) and $S\left(t_{p}\right)^{3}$ is the same on the two sheets $\mathcal{D}_{210}, \mathcal{D}_{120}$, so again $X$ reduces to a single sheet. Similarly, so do $Y$ and $Z$.

As a result, $S\left(t_{p}\right)^{3}$ is uniquely determined if we know $z_{p}$ and $w_{p}$. In fact on any Riemann sheet $(m, n)$ the analytic continuation of $S\left(t_{p}\right)^{3}$ is

$$
\left(y_{p} / x_{p}\right)^{3 r} S_{0}\left(t_{p}\right)^{3}
$$


where

$$
r= \begin{cases}(m+n) / 3 & \text { on even sheets, } \\ (m+n-1) / 3 & \text { on odd sheets. }\end{cases}
$$

The $x_{p}, y_{p}$ in (5.10) are those of the sheet under consideration.

It therefore makes sense to look for a single-valued meromorphic function of $z_{p}$ and $w_{p}$ that is equal to $S\left(t_{p}\right)^{3}$. We can write $S\left(t_{p}\right)$ itself as $S\left(z_{p}, w_{p}\right)$ provided we accept that it is three-valued in the rather trivial way that its values differ by factors of $\omega$. Then from the automorphisms (3.4) and (5.5), this function must satisfy the three relations

$$
S\left(z_{p}, w_{p}\right)=\left(x_{p} / y_{p}\right) S\left(x^{-1} z_{p}^{-1}, x^{-1} w_{p}^{-1}\right)=S\left(x z_{p}^{-1}, w_{p}^{-1}\right)=S\left(z_{p}^{-1}, x w_{p}^{-1}\right)
$$

for all $z_{p}, w_{p}$. Also, using (2.2) and (5.4), the relation (2.7) becomes

$$
S\left(z_{p}, w_{p}\right) S\left(-1 / w_{p}, z_{p} / w_{p}\right) S\left(-w_{p} / z_{p},-1 / z_{p}\right)=k^{-1 / 3} x_{p} .
$$

The function $x_{p}^{-1}$ has no zeros or poles on even sheets, whereas on odd sheets (where $y_{p} \simeq \omega^{i}$ ) it has the same zeros and poles as $y_{p} / x_{p}$. It is therefore useful to work not with $S\left(z_{p}, w_{p}\right)^{3}$, but the function

$$
\tilde{S}\left(z_{p}, w_{p}\right)=x_{p}^{-1} S\left(z_{p}, w_{p}\right)^{3},
$$

since from (5.10) and (5.11) this has the same poles and zeros as $\left(y_{p} / x_{p}\right)^{m+n}$ on all Riemann sheets $(m, n)$, even and odd. From (5.2) we can write $\left(y_{p} / x_{p}\right)$ as a function of either $z_{p}, w_{p}$ or $z_{p} / w_{p}$ : this suggests that it may be possible to write $\tilde{S}\left(z_{p}, w_{p}\right)$ as a product of functions of these individual variables. We do this in the next section.

\section{6. $S\left(t_{p}\right)$ as a function of $z_{p}, w_{p}$}

Define the functions

$$
F(z)=\prod_{j=1}^{\infty} \frac{\left(1-x^{j} z\right)^{j}}{\left(1-x^{j} z^{-1}\right)^{j}} \quad \text { and } \quad G(z)=\frac{F(\omega z)}{F\left(\omega^{2} z\right)},
$$

so $F(z)=1 / F\left(z^{-1}\right), G(z)=G\left(z^{-1}\right)$ and

$$
G(z) / G(x z)=\omega h(z)
$$

for all complex numbers $z$.

Consider the product

$$
P=G\left(z_{p}\right)^{\alpha} G\left(-1 / w_{p}\right)^{\beta} G\left(-w_{p} / z_{p}\right)^{\gamma}
$$


for arbitrary integers $\alpha, \beta, \gamma$. As a first step, we ask if we can choose $\alpha, \beta, \gamma$ so that $P$ has the same poles and zeros as $\tilde{S}\left(z_{p}, w_{p}\right)$, that is, as $\left(y_{p} / x_{p}\right)^{m+n}$ on all sheets $(m, n)$.

The integers $m, n$ specify a sheet and are defined in (5.6). From (5.2), on sheet $(m, n)$ the function $y_{p} / x_{p}$ has a simple zero when $z_{p}=x^{m} \omega^{2}, w_{p}=-x^{n} \omega, z_{p} / w_{p}=$ $-\omega^{m-n} \omega$. Any one of these equalities implies the other two, so at this point $\tilde{S}\left(z_{p}, w_{p}\right)$ has a zero of order $m+n$. On the other hand, the three factors of $P$ have zeros of order $-m \alpha, n \beta,(m-n) \gamma$, respectively. Thus we require $m+n=-m \alpha+n \beta+(m-n) \gamma$, for all allowed integers $m, n$ and fixed values of $\alpha, \beta, \gamma$. This will be so if and only if

$$
\alpha=\gamma-1, \quad \beta=\gamma+1
$$

There are also possible zeros and poles when $z_{p}=x^{m} \omega, w_{p}=-x^{n} \omega^{2}, z_{p} / w_{p}=$ $-\omega^{m-n} \omega^{2}$, but the only difference from the above is that all the orders are negated, so again we obtain the conditions (6.4).

Neither $\tilde{S}\left(z_{p}, w_{p}\right)$ not $P$ has any other other zeros or poles, and $\tilde{S}\left(z_{p}, w_{p}\right)$ is independent of the integer $\gamma$, which is still arbitrary. Substituting (6.4) into (6.3), this implies that the functions

$$
\tilde{S}\left(z_{p}, w_{p}\right) G\left(z_{p}\right) / G\left(-1 / w_{p}\right), \quad G\left(z_{p}\right) G\left(-1 / w_{p}\right) G\left(-w_{p} / z_{p}\right)
$$

have no zeros or poles anywhere on the Riemann surface. Using (5.8) and (5.14), they have values $k^{-1 / 3}, F(\omega)^{-3}$ at $p=p(1)$, so if they were constants it would follow that

$$
\begin{gathered}
\tilde{S}\left(z_{p}, w_{p}\right)=k^{-1 / 3} G\left(-1 / w_{p}\right) / G\left(z_{p}\right), \\
G\left(z_{p}\right) G\left(-1 / w_{p}\right) G\left(-w_{p} / z_{p}\right)=1 / F(\omega)^{3} .
\end{gathered}
$$

We can prove that these relations are indeed true by using Liouville's theorem for a single Riemann sheet. Let $T\left(z_{p}, w_{p}\right)$ be the ratio of the RHS of (5.14) to the RHS of (6.5). Then from (5.12) and (6.1)-(6.2) it follows that

$$
T\left(z_{p}, w_{p}\right)=T\left(x^{-1} z_{p}^{-1}, x^{-1} w_{p}^{-1}\right)=T\left(x z_{p}^{-1}, w_{p}^{-1}\right)=T\left(z_{p}^{-1}, x w_{p}^{-1}\right) .
$$

Thus the function $T$ is unchanged by the three automorphisms $A_{0}, A_{1}, A_{2}$. It is therefore a single-valued function of the variable $t_{p}$, without the branch cuts of Figure 2 . It has no zeros or poles in $\mathcal{D}$, so it has no zeros or poles in the complex $t_{p}$ plane, including the point at infinity. By Liouville's theorem it is therefore a constant. It is unity when $z_{p}=\omega^{2}$ or $\omega$, that is, when $t_{p}=0$ or $\infty$, so it is one. This proves the identity (6.5). The identity (6.6) can be proved similarly by taking $T\left(z_{p}, w_{p}\right)$ to be the ratio of of the LHS of (6.6) to the RHS. 
Two more identities We originally tried a much more general ansatz for the product $P$, allowing for factors such as $1-x^{j} z$ raised to a power linear in $j$ and mod $(j, 3)$. As a result we discovered yet two more identities satisfied by $z_{p}, w_{p}$. Define the function

$$
\tilde{h}(z)=\prod_{n=1}^{\infty} \frac{\left(1-x^{3 n-2} \omega z\right)\left(1-x^{3 n-1} \omega^{2} / z\right)}{\left(1-x^{3 n-2} \omega^{2} z\right)\left(1-x^{3 n-1} \omega / z\right)},
$$

then we find that

$$
\tilde{h}\left(z_{p}\right) \tilde{h}\left(-w_{p}\right)=\tilde{h}\left(-1 / w_{p}\right) \tilde{h}\left(-z_{p} / w_{p}\right)=\tilde{h}\left(-w_{p} / z_{p}\right) \tilde{h}\left(1 / z_{p}\right) .
$$

We can prove these identities in a similar way. First note that

$$
\tilde{h}\left(\frac{x}{z}\right)=\frac{1}{\tilde{h}(z)}, \quad \tilde{h}\left(\frac{z}{x}\right)=\frac{\omega^{2}}{\tilde{h}\left(x^{-1} z^{-1}\right)} \quad \text { and } \quad h(z)=\tilde{h}\left(\frac{z}{x}\right) \tilde{h}(z) \tilde{h}\left(\frac{1}{z}\right)
$$

for all $z$. We can use this last formula and (5.2) to eliminate ratios such as

$$
\frac{\tilde{h}\left(-x^{-1} w^{-1}\right)}{\tilde{h}\left(x^{-1} z\right)}
$$

in favour of $\tilde{h}$ functions whose arguments do not contain $x^{-1}$ as a factor. Using this fact and applying the automorphisms $A_{0}, A_{1}, A_{2}$ to the ratios of the expressions in (6.7), we find that the automorphisms merely permute these ratios. If we write the three expressions as $J_{1}, J_{2}, J_{3}$ and form, for arbitrary $\alpha$,

$$
T\left(z_{p}, w_{p}\right)=\left(\alpha-\frac{J_{2}}{J_{1}}\right)\left(\alpha-\frac{J_{3}}{J_{2}}\right)\left(\alpha-\frac{J_{1}}{J_{3}}\right)\left(\alpha-\frac{J_{1}}{J_{2}}\right)\left(\alpha-\frac{J_{2}}{J_{3}}\right)\left(\alpha-\frac{J_{3}}{J_{1}}\right),
$$

then this $T\left(z_{p}, w_{p}\right)$ is unchanged by the automorphisms, so is a single-valued function of $t_{p}$. It has no zeros or poles in $\mathcal{D}$, so by Liouville's theorem it is a constant. At $p=p(1)$ or $p(2)$, the $J_{i}$ 's are equal, so for all $z_{p}, w_{p}$

$$
T\left(z_{p}, w_{p}\right)=(\alpha-1)^{6} .
$$

It follows that $J_{1}=J_{2}=J_{3}$ for all $t_{p}$, which establishes the identities (6.7).

For an arbitrarly chosen numerical value of $z_{p}$, working to 32 digits of accuracy, we have successfully checked the identities (6.5)-(6.7) to twenty terms in an expansion in powers of $x$.

From (2.2) and (5.4), the map $p \rightarrow R S V p$ takes $x_{p}, t_{p}, z_{p}, w_{p}$ to $1 / x_{p}, 1 / t_{p},-w_{p}$, $-z_{p}$. From (5.14) and (6.5), noting that $G\left(-1 / w_{p}\right)=G\left(-w_{p}\right)$, it is apparent that

$$
S_{p} S_{R S V_{p}}=S\left(z_{p}, w_{p}\right) S\left(-w_{p},-z_{p}\right)=k^{-2 / 9},
$$

in agreement with (2.9). 


\section{Summary}

For the case when $N=3$ and $q$ is related to $p$ by (2.4), the generalised order parameter function $G_{p q}(r)$ is given by (2.5), where $x_{p}^{-1} S\left(t_{p}\right)^{3}$ can be simply expressed by (5.14) and (6.5) as a product of functions of the hyperelliptic variables $z_{p}, w_{p}$ of Section 5. This is the first time that a thermodynamic property of the $N>2$ chiral Potts model has been so expressed. (As distinct from algebraic functions of the Boltzmann weights, such as the function of $f_{p q}$ of [12].)

The functions $F(z), G(z)$ in (6.1) are infinite products similar to elliptic functions, except that factors such as $1-x^{j} z$ are raised to the power $j$. Such extensions of elliptic functions occur in the free energies of other solvable models, notably the Ising model. Good examples are [13, Equations (B.10), (B.17) and (B.18)].

We emphasise that (5.14) and (6.5) are in terms of the alternative hyperelliptic parametrisation of Section 5 herein. Papers [8] to [16] are in terms of the original hyperelliptic parametrisation of Section 4.

It is still an interesting question whether $G_{p q}(r)$ can be simply expressed as a function of such variables for arbitrary $p, q$. The result of this paper implies that one must use the hyperelliptic parametrisation of Section 5, rather than that of Section 4. There is a difficulty with this: if we write $R^{6} p, R^{6} q$ as $p^{\prime}, q^{\prime}$, the relations (5.4) imply that $z_{p^{\prime}}=z_{p}$ and $w_{p^{\prime}}=w_{p}$, whereas it is not true that $G_{p^{\prime} . q}(r)=G_{p q}(r)$ or that $G_{p, q^{\prime}}(r)=G_{p q}(r)$. This means that $G_{p q}(r)$ cannot be a single-valued function of $z_{p}, w_{p}, z_{q}, w_{q}$. However, the function $L_{p q}(r)=G_{p q}(r) G_{R q, R p}(r)$ of $[11]$ is unchanged by $p \rightarrow p^{\prime}$, and by $q \rightarrow q^{\prime}$, so may be so expressible.

\section{Appendix A.}

Here we show how the alternative hyperelliptic parametrisation of Section 5 can be obtained from the original parametrisation of Section 4 by a simple mapping.

Let $k, k^{\prime}, x_{p}, y_{p}, \mu_{p}, t_{p}$ be the variables of Section 4 . Define new variables $\hat{k}, \hat{k}^{\prime}$, $\hat{x}_{p}, \hat{y}_{p}, \hat{\mu}_{p}, \hat{t}_{p}$ so that

$$
\begin{gathered}
k=\hat{k}^{-1}, \quad k^{\prime}=i \hat{k}^{\prime} / \hat{k}, \quad x_{p}=1 / \hat{x}_{p}, \quad y_{p}=\hat{y}_{p}, \\
\mu_{p}=\mathrm{e}^{-\mathrm{i} \pi / 2 N} \hat{x}_{p} \hat{\mu}_{p}, \quad y_{p} / x_{p}=\hat{t}_{p} .
\end{gathered}
$$

Leave $x, z_{p}, w_{p}$ and the functions $h(z), \phi(z)$ unchanged.

Then the relations $(1.1),(2.1)$ remain satisfied if we replace $k, k^{\prime}, x_{p}, y_{p}, \mu_{p}, t_{p}$ therein by $\hat{k}, \hat{k}^{\prime}, \hat{x}_{p}, \hat{y}_{p}, \hat{\mu}_{p}, \hat{t}_{p}$. The relations (4.1), (4.4), (4.5) become

$$
-\hat{k}^{\prime 2}=27 x \prod_{n=1}^{\infty}\left(\frac{1-x^{3 n}}{1-x^{n}}\right)^{12} \text {, }
$$




$$
\begin{gathered}
\hat{y}_{p} / \hat{x}_{p}=\omega h\left(z_{p}\right)=h\left(-1 / w_{p}\right)=\omega^{2} h\left(-w_{p} / z_{p}, x\right) \\
-\hat{x}_{p}^{-3} \hat{y}_{p}^{3} \hat{\mu}_{p}^{-6}=\phi\left(x z_{p} / w_{p}^{2}\right)^{3}=\phi\left(-x z_{p} w_{p}\right)^{3}=\phi\left(-x w_{p} / z_{p}^{2}\right)^{3} .
\end{gathered}
$$

Define automorphisms $\hat{R}, \hat{S}, \hat{V}, \hat{M}$ by (2.2) with $x_{p}, y_{p}, \ldots, M_{p}$ replaced by $\hat{x}_{p}, \hat{y}_{p}, \ldots, \hat{M}_{p}$. Then

$$
\hat{R}=V S, \quad \hat{S}=M^{-1} V^{-1} R, \quad \hat{V}=V, \quad \hat{M}=M
$$

and the relations (2.3) remain satisfied if $R, S, V, M$ therein are replaced by $\hat{R}, \hat{S}, \hat{V}$, $\hat{M}$. From (4.7) it follows that

$$
\begin{aligned}
& z_{\hat{R}_{p}}=-x w_{p}, \quad z_{\hat{s}_{p}}=-1 /\left(x w_{p}\right), \quad z_{\hat{v}_{p}}=-1 / w_{p}, \\
& w_{\hat{R} p}=w_{p} / z_{p}, \quad w_{\hat{S}_{p}}=-1 /\left(x z_{p}\right), \quad w_{\hat{v}_{p}}=z_{p} / w_{p} .
\end{aligned}
$$

Now drop the hats on $\hat{k}, \hat{k}^{\prime}, \hat{x}_{p}, \ldots, \hat{M}$ to obtain (5.1)-(5.4). Equations (1.1), (2.1), (2.2) remain true.

\section{References}

[1] G. Albertini, B. M. McCoy, J. H. H. Perk and S. Tang, "Excitation spectrum and order parameter for the integrable $N$-state chiral Potts model", Nuc. Phys. B 314 (1989) 741-763.

[2] H. Au-Yang and J. H. H. Perk, "Onsager's star-triangle equation: master key to integrability", Adv. Stud. Pure Math. 19 (1989) 57-94.

[3] R. J. Baxter, "Solvable eight-vertex model on an arbitrary planar lattice", Phil. Trans. Roy. Soc. (London) 289 (1978) 315-346.

[4] R. J. Baxter, "Corner transfer matrices", Physica A (1981) 18-27.

[5] R. J. Baxter, Exactly Solved Models in Statistical Mechanics (Academic, London, 1982).

[6] R. J. Baxter, "Free energy of the solvable chiral Potts model", J. Stat. Phys. 52 (1988) 639-667.

[7] R. J. Baxter, "Chiral Potts model: eigenvalues of the transfer matrix", Phys. Lett. A 146 (1990) 110-114.

[8] R. J. Baxter, "Hyperelliptic function parametrization for the chiral Potts model", in Proc. Intnl. Conf. Marhematicians, Kyoto 1990, (Springer, Tokyo, 1991) 1305-1317.

[9] R. J. Baxter, "Corner transfer matrices of the chiral Potts model. II. The triangular lattice.", J. Stat. Phys. 70 (1993) 535-582.

[10] R. J. Baxter, "Elliptic parametrization of the three-state chiral Potts model", in Integrable Quantum Field Theories (ed. L. Bonora et al.), (Plenum Press, New York, 1993) 27-37.

[11] R. J. Baxter, "Functional relations for the order parameters of the chiral Potts model", J. Stat. Phys. 91 (1998) 499-524.

[12] R. J. Baxter, "Some hyperelliptic function identities that occur in the chiral Potts model", J. Phys. A 31 (1998) 6807-6818.

[13] R. J. Baxter, "The 'inversion relation" method for obtaining the free energy of the chiral Potts model". Physica A 322 (2003) 407-431.

[14] R. J. Baxter, "Derivation of the order parameter of the chiral Potts model", Phys. Rev. Lett. 94 (2005) 1.30602. 
[15] R. J. Baxter, "The order parameter of the chiral Potts model", J. Stat. Phys. 120 (2005) 1-36.

[16] R. J. Baxter, "The challenge of the chiral Potts model", J. Physics: Conference Ser. (2006) to appear.

[17] R. J. Baxter, J. H. H. Perk and H. Au-Yang, "New solutions of the star-triangle relations for the chiral Potts model", Phys. Lett. A 128 (1988) 138-142.

[18] M. Jimbo, T. Miwa and A. Nakayashiki, "Difference equations for the correlation functions of the eight-vertex model", J. Phys. A 26 (1993) 2199-2210.

[19] L. Onsager, "Crystal statistics. I. A two-dimensional model with an order-disorder transition", Phys. Rev. 65 (1944) 117-49.

[20] C. N. Yang, "The spontaneous magnetization of a two-dimensional Ising model", Phys. Rev. 85 (1952) 808-816. 